\title{
I farmaci "generici" in Italia: opportunità di ricerca \\ e sviluppo di prodotti di qualità \\ a prezzi competitivi
}

REVIEW

Mario Eandi*, Carlo Della Pepa*

\begin{abstract}
Every drug whose copyright coverage has expired can be manufactured by a pharmaceutical company other than the one that developed it, giving rise to the market of the so-called generic drugs. It can be distinguished among two types of generic drugs: branded and unbranded, according to the choice of the manufacturer to assign it a fantasy name or to sell it with the name of the active principle, followed by the company's name. In order to be accepted for marketing, every new drug has to pass the registration procedures of the Italian Ministry of Health, which are simplified for generics, as it's sufficient to demonstrate the bioequivalence, considered a reliable proxy of therapeutic equivalence, of the new drug with the standard formulations it copies. The bioequivalence of two drugs depends on various parameters, related to the quality of the raw materials employed and the industrial processes they go through. There are several ways of assessing the equivalence between pharmaceutical products, and it is important the registration studies are conducted following strict rules, in order to guarantee the quality of generics, fundamental for achieving the trust of health operators and patients.

The reason to be of generic drugs is essentially economic, as they bare no therapeutic innovation, and their introduction is mainly aimed to contain drug expenditures and to facilitate a competitive market, but to do so, they need to be widely accepted by the medical community and the population.

In Italy, the market of generic drugs is very young and relatively underdeveloped if compared to what is observed in other industrialized countries such as the USA, Germany or UK, but it has a good expansion potential, as it also represents a chance for quality enhancement for the Italian pharmaceutical industry.
\end{abstract}

Farmeconomia e percorsi terapeutici 2003; 4 (2): $65-76$

\section{INTRODUZIONE}

Ogni prodotto farmaceutico, allo scadere della protezione brevettuale sulla materia prima, può essere copiato, riprodotto e commercializzato da ditte farmaceutiche concorrenti, alimentando il mercato dei cosiddetti "generici".

I prodotti "generici" sono copie di un prodotto farmaceutico maturo (detto anche "innovatore") e come questo sono specialità farmaceutiche a tutti gli effetti, che ottengono l'autorizzazione all'immissione in commercio (AIC), ossia la registrazione, dal Ministero della Salute secondo procedure abbreviate. Infatti, per registrare un "generico" è sufficiente presentare al Ministero della Salute una domanda di AIC, accompagnata dalla documentazione del suo sviluppo galenico e dalla dimostrazione della sua equivalenza farmaceutica e, quan- do richiesto, della sua bioequivalenza con il prodotto "innovatore" copiato.

I prodotti farmaceutici "copia” possono essere registrati e commercializzati con un nome di fantasia ("generici branded", non distinguibili da una qualsiasi altra specialità medicinale) 0 con la denominazione comune internazionale (DCI) seguita dal nome del produttore ("generici unbranded" o semplicemente "generici").

Il mercato dei "generici unbranded", alimentato dalla scadenza del brevetto di un numero crescente di molecole importanti e di uso consolidato nonché da una specifica regolamentazione favorevole, è un fenomeno consolidato in alcuni Paesi industrializzati, quali gli Stati Uniti, il Regno Unito e la Germania.

In Italia, prima dell'introduzione del brevetto sui farmaci alla fine degli anni ' 70 , si era sviluppato esclusivamente il mercato dei "generici branded" ossia di specialità copia con
* Farmacologia Clinica Università di Torino 
nome di fantasia. Successivamente, il fenomeno del co-marketing aveva consentito il moltiplicarsi (fino a tre) di prodotti innovatori assolutamente identici per formulazione e fabbricazione, ma venduti con differenti nomi di fantasia. In questo caso non si può parlare di generici branded, ma di prodotti uguali registrati ciascuno con un differente marchio. Alcune innovazioni normative, introdotte negli ultimi anni e mirate soprattutto a razionalizzare il mercato farmaceutico e contenerne la spesa a carico del SSN, hanno creato le condizioni necessarie per favorire lo sviluppo del mercato dei "generici" propriamente detti, anche in Italia.

Cardini della politica dei "generici" in Italia sono essenzialmente tre: 1) il prezzo di un "generico" non può essere superiore all' $80 \%$ del prezzo del prodotto "innovatore", mentre un produttore può fissarlo ad un livello anche notevolmente inferiore, innescando in tal modo una dinamica di prezzi al ribasso grazie alla possibilità di entrare in un mercato concorrenziale; 2) il prezzo del generico, o il prezzo più basso tra quelli dei prodotti generici equivalenti, viene assunto come prezzo di riferimento rimborsabile dal SSN per quel farmaco; 3) il farmacista può, anzi deve, dispensare un "generico" in sostituzione di un equivalente prodotto di marchio, salvo il caso in cui il medico specifichi il contrario o il paziente sia disposto a pagare l'eventuale differenza rispetto alla quota rimborsata dal SSN.

Una campagna pubblicistica, indirizzata alla popolazione e alla classe medica, ha cercato di creare un clima favorevole all' accettazione dei generici.

Il mercato dei generici, nell' anno e mezzo trascorso dalla loro ufficiale introduzione in Italia, ha raggiunto quote sensibili, ma non straordinarie: nel 2002 la quota di mercato dei generici è stata pari solo al 2,2\% (34,1 milioni di confezioni) del totale in unità di pezzi venduti per un valore pari all' 1,3\% (146,8 milioni di euro) della spesa farmaceutica nazionale.

D'altra parte, nella popolazione, oltre che tra gli operatori sanitari, su questa categoria di farmaci permangono diversi dubbi e perplessità, che vorremmo contribuire a chiarire con queste brevi considerazioni tecniche.

\section{IGENERICI: \\ FARMACIESSENZIALMENTESIMILI ALL'INNOVATORE}

I farmaci "generici" sono stati pubblicizzati come farmaci "uguali" a quelli di marchio già in commercio ma "più convenienti" perché venduti ad un prezzo inferiore: il loro utilizzo consente di ottenere significativi risparmi a vantaggio del SSN e del cittadino senza uno scadimento dei risultati terapeutici.
In realtà, i farmaci "generici" non sono mai perfettamente uguali al prodotto che imitano, ma devono essere "essenzialmente simili" a questo.

Una specialità medicinale viene considerata "essenzialmente simile" ad un'altra se ha la stessa composizione quali-quantitativa in principi attivi e la stessa forma farmaceutica e se viene dimostrata la sua bioequivalenza.

In altri termini, sotto il profilo tecnico un farmaco "generico" è contemporaneamente un "equivalente farmaceutico" ed un "equivalente terapeutico" della specialità medicinale di marchio imitata.

Infatti, il farmaco "generico" ha la stessa composizione in principi attivi, una forma farmaceutica equivalente, lo stesso dosaggio e la stessa via di somministrazione del prodotto innovatore. Quindi, per quanto riguarda la forma farmaceutica, un prodotto "generico" rientra nella categoria degli "equivalenti farmaceutici".

La formulazione di un equivalente farmaceutico del prodotto innovatore è la prima tappa fondamentale, spesso l'unica, del processo di ricerca e sviluppo industriale di un nuovo generico.

Nello sviluppo galenico di un farmaco generico un'azienda farmaceutica può esprimere al meglio il proprio know-how tecnologico e industriale e attraverso la produzione di "generici” di alta qualità può non solo conquistare crescenti quote di mercato, ma anche contribuire a creare un clima di fiducia verso il farmaco generico nel medico prescrittore e nel paziente consumatore.

La scelta delle materie prime da utilizzare è una delle tappe più qualificanti per imprimere al prodotto finito i caratteri di un prodotto farmaceutico di elevato standard qualitativo e di sicura affidabilità. Tuttavia, nell' acquisto delle materie prime il produttore di generici può realizzare sensibili risparmi, soprattutto se rinuncia a standard qualitativi elevati.

Le materie prime, principi attivi ed eccipienti, utilizzate per formulare e produrre un "generico", pur rispettando i parametri di qualità previsti dalla Farmacopea Ufficiale e dalle norme che regolano la registrazione dei prodotti medicinali, possono presentare sensibili differenze, rispetto a quelle utilizzate per produrre il farmaco originale copiato, per quanto riguarda il profilo quali-quantitativo delle impurezze ammesse e le caratteristiche fisiche e chimico-fisiche, quali granulometria, polimorfismo, solubilità.

D'altra parte una stessa forma farmaceutica (es. compressa) può essere prodotta scegliendo tra un'ampia gamma di eccipienti ed utilizzando differenti tecnologie e processi di fabbricazione. 


\begin{tabular}{|c|c|c|}
\hline \multirow{2}{*}{$\begin{array}{l}\text { Forma farmaceutica } \\
\text { Proprietà }\end{array}$} & \multicolumn{2}{|c|}{ Eccipiente } \\
\hline & Parametro & Meccanismo \\
\hline Stabilità & $\begin{array}{l}\text { Umidità residua } \\
\text { Adsorbimento di umidità }\end{array}$ & $\begin{array}{l}\text { Protezione del principio attivo } \\
\text { dalla degradazione idrolitica }\end{array}$ \\
\hline \multirow[t]{3}{*}{ Processo di fabbricazione } & $\begin{array}{l}\text { Area di superficie } \\
\text { Energia libera di superficie } \\
\text { Difetti di cristallizzazione } \\
\text { Potere deformante }\end{array}$ & $\begin{array}{l}\text { Influiscono sulla } \\
\text { comprimibilità e sulla } \\
\text { possibilità di produrre con } \\
\text { successo compresse mediante } \\
\text { macchine comprimitrici ad } \\
\text { alta velocità }\end{array}$ \\
\hline & $\begin{array}{l}\text { Distribuzione della dimensione } \\
\text { delle particelle } \\
\text { Forma delle particelle }\end{array}$ & $\begin{array}{l}\text { Influiscono sulla fluidità, } \\
\text { sull'efficienza della } \\
\text { miscelazione a secco e sul } \\
\text { potere segregante }\end{array}$ \\
\hline & $\begin{array}{l}\text { Comprimibilità } \\
\text { Fluidità } \\
\text { Potere diluente }\end{array}$ & $\begin{array}{l}\text { Condizionano la possibilità di } \\
\text { scegliere la compressione } \\
\text { diretta come metodo di } \\
\text { fabbricazione delle compresse }\end{array}$ \\
\hline Performance & $\begin{array}{l}\text { Adesività } \\
\text { Coesività } \\
\text { Energia libera di superficie } \\
\text { Grado di igroscopicità }\end{array}$ & $\begin{array}{l}\text { Condizionano i processi di } \\
\text { disintegrazione del formulato } \\
\text { e di solubilizzazione del } \\
\text { farmaco }\end{array}$ \\
\hline
\end{tabular}

La messa a punto di nuovi prodotti generici, che possano competere come livello qualitativo e come costi di produzione con $\mathrm{i}$ relativi prodotti innovatori, può richiedere lo sviluppo di tecnologie farmaceutiche non banali. Nel campo delle forme solide e delle matrici a rilascio controllato, le tecnologie farma- ceutiche e l'uso di eccipienti ad alta funzionalità hanno raggiunto livelli notevoli di complessità.

Il ruolo funzionale degli eccipienti è fondamentale per caratterizzare una forma solida. La tabella 1 riassume l'importanza dei diversi parametri caratterizzanti un eccipiente ed il loro

\section{Tabella 1}

Parametri specifici di un eccipiente e relativi ruoli o meccanismi nel determinare le proprietà di una forma farmaceutica solida

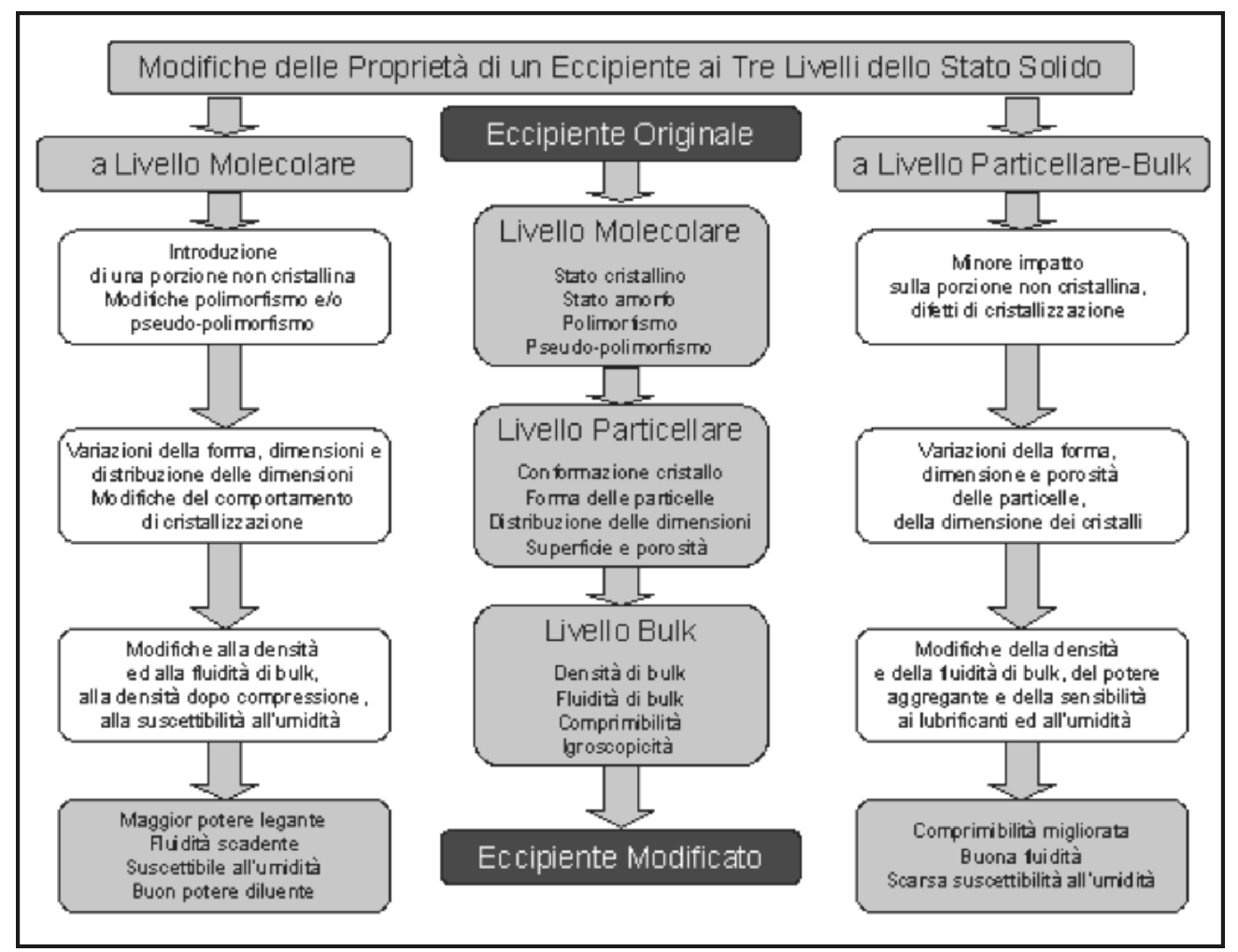

\section{Figura 1}

I tre livelli dello stato solido di un eccipiente e possibili

modificazioni delle sue proprietà intervenendo a livello molecolare o a livello particellare e bulk 
impatto su tre proprietà generali di una forma farmaceutica: la stabilità, il processo di fabbricazione e la performance.

Conoscendo approfonditamente i vantaggi e i limiti di ciascun eccipiente, il tecnico farmaceutico può ridurre i tempi e i costi dello sviluppo galenico, della fase di scale-up e di avvio della produzione industriale.

Negli ultimi decenni la disponibilità di nuovi eccipienti e il miglioramento qualitativo di quelli da tempo esistenti hanno consentito di introdurre processi di fabbricazione più efficienti, più rapidi e meno costosi, come la compressione diretta.

In realtà, pochi sono stati i nuovi eccipienti introdotti sul mercato negli ultimi decenni. Ciò è dovuto agli alti costi e ai tempi molto lunghi richiesti per far approvare un nuovo eccipiente, fattori che finiscono per rendere poco remunerativo e molto rischioso lo sviluppo di un nuovo eccipiente, analogamente a quanto avviene con i farmaci innovativi.

I produttori di eccipienti hanno preferito l'opzione di migliorare il grado di quelli già esistenti e di studiare nuove combinazioni caratterizzate da elevate funzionalità.

La figura 1 rappresenta i tre livelli dello stato solido di un eccipiente (molecolare, particellare e bulk), i parametri caratteristici di ciascun livello, l'interdipendenza tra loro e le modificazioni funzionali che si possono ottenere introducendo modifiche a livello molecolare o a livello particellare-bulk.

Le caratteristiche che differenziano una sostanza solida a livello molecolare sono lo stato cristallino o amorfo e la presenza di polimorfismo o di pseudo-polimorfismi. A livello particellare i parametri caratterizzanti una sostanza solida sono la forma, la dimensione e la distribuzione delle dimensioni delle particelle, il tipo di cristallizzazione, la porosità delle particelle e della superficie. Infine a livello di massa (bulk), le caratteristiche di un eccipiente

\section{Proprietà delle particelle}

Aumento delle dimensioni

Riduzione della variabilità della dimensione

Aumento della porosità delle particelle

Formazione di superfici porose

Potere diluente

\section{Tabella 2}

Relazione tra alcune proprietà fisiche delle particelle e funzionalità dell'eccipiente solido sono rappresentate dalla densità e fluidità di bulk, dalla comprimibilità e dalla igroscopicità.

Introducendo una porzione non cristallina e varianti polimorfiche o pseudo-polimorfiche a livello molecolare si può migliorare la capacità di legame ed il potere diluente di un eccipiente, peggiorando, tuttavia la fluidità e conservando un certo livello di igroscopicità.

Intervenendo soprattutto a livello particellare, si può migliorare la comprimibilità e la fluidità dell' eccipiente e ridurre la sua sensibilità all'umidità.

La tabella 2 indica come la modificazione di alcune caratteristiche fisiche delle particelle possa contribuire a modificare la funzionalità di un eccipiente.

L'industria farmaceutica che si dedica al mercato dei generici ha un estremo bisogno di eccipienti ad alta efficienza che possano facilitare lo sviluppo di nuovi prodotti, qualitativamente comparabili e bioequivalenti con i relativi prodotti innovatori. Gli eccipienti ad alta funzionalità, grazie alla loro elevata flessibilità ed alla capacità di modulare un ampio spettro di proprietà biofarmaceutiche, sono preziosi strumenti per i produttori di generici di alta qualità.

Gli eccipienti moderni ad alta efficienza vengono sempre più frequentemente utilizzati nelle formulazioni dei prodotti generici perché rappresentano uno dei più importanti fattori capaci di garantire successo e remunerazione al produttore: alti livelli qualitativi del medicinale, completo rispetto delle norme regolatorie, equivalenza farmaceutica con i prodotti innovatori spesso connotati come prodotti ad alta tecnologia farmaceutica, facilitazione nel raggiungimento della bioequivalenza durante la fase di sviluppo galenico, tempi rapidi di sviluppo, costi ridotti di sviluppo e di produzione.

Queste poche e sintetiche osservazioni sottolineano non solo l'elevato contenuto tecnologico presente nella maggior parte dei prodotti generici oggi in circolazione, ma anche il fatto che l'innovazione tecnologica, anche in questo campo merceologico, rappresenta uno dei principali motori per conquistare quote di mercato e ottenere buoni profitti.

\section{PROFILOBIOFARMACEUTICO DIUNGENERICO: BIODISPONIBILITÀ SISTEMICAEBIOEQUIVALENZA}

L'insieme delle variabili chimico-fisiche di una formulazione farmaceutica sono alla base del peculiare comportamento biofarmaceutico di ogni prodotto medicinale, descritto, nel caso 
di forme solide, dal profilo di rilascio "in vitro" e soprattutto dal profilo di biodisponibilità "in vivo".

Le caratteristiche chimico-fisiche delle materie prime e le tecnologie farmaceutiche utilizzate per preparate una specifica forma farmaceutica condizionano la velocità di passaggio in soluzione del principio attivo e, nel caso di forme solide, anche la velocità di disgregazione.

Due prodotti costituiti da una forma solida che siano "equivalenti farmaceutici" dovrebbero avere un profilo di rilascio "in vitro" sovrapponibile come valore medio, con margini di variabilità minima accettabile. Lo studio della funzione di rilascio in vitro è importante per definire la qualità di una nuova formulazione farmaceutica (ad esempio di un generico) sia in termini assoluti sia in termini relativi ad un eventuale prodotto standard (es. innovatore da copiare).

La velocità di rilascio "in vitro", tuttavia, non sempre è un buon indicatore della velocità di rilascio in vivo e soprattutto non sempre correla in modo predittivo con la biodisponibilità sistemica. I processi di disgregazione della forma solida e di solubilizzazione, quando avvengono in vivo nella zona di applicazione del farmaco (es. a livello gastrointestinale), possono costituire fattori limitanti la velocità di assorbimento e la quantità di farmaco assorbito e perciò condizionare la biodisponibilità sistemica del principio attivo.

La correlazione "in vitro-in vivo" può essere determinata solo a posteriori, ma, quando sia stata definita, può talvolta essere utilizzata per evitare ulteriori studi di biodisponibilità e di bioequivalenza su soggetti umani.

Purtroppo, nella maggior parte dei casi lo studio della correlazione in vitro-in vivo non è attuabile o non produce risultati affidabili.

Pertanto, per caratterizzare il profilo biofarmaceutico di una nuova formulazione solida, il più delle volte accanto allo studio delle curve di rilascio in vitro, è necessario effettuare studi di biodisponibilità in vivo e testare direttamente la bioequivalenza della nuova formulazione con quella di riferimento.

Per biodisponibilità s'intende la quantità e la velocità con cui la sostanza attiva o la frazione terapeutica viene assorbita da una forma farmaceutica e risulta disponibile nella sede d'azione [1].

In realtà, nel definire la biodisponibilità di un prodotto si assume che la curva delle concentrazioni plasmatiche del farmaco rappresenti in modo adeguato la disponibilità del principio attivo nella sede d'azione. Quindi, la biodisponibilità sistemica di un prodotto viene valutata dal profilo della curva concentra- zione-tempo del principio attivo, utilizzando il parametro AUC (area sotto la curva), come indicatore della quantità di farmaco reso biodisponibile, e i parametri Cmax (concentrazione di picco massimo) e tmax (tempo di picco massimo) come indicatori della velocità con cui il principio attivo è reso disponibile.

Fatta eccezione per le formulazioni costituite da soluzioni pronte per uso parenterale od orale, che per definizione hanno la stessa biodisponibilità sistemica dei relativi prodotti standard, tutti gli altri prodotti costituiti da forme farmaceutiche solide o da altre matrici complesse, pur essendo essenzialmente simili al prodotto che imitano, possono avere una biodisponibilità sistemica significativamente differente e comportare quindi un differente profilo di efficacia e tollerabilità.

Un prodotto "generico", per definizione, deve avere non solo la stessa composizione quali-quantitativa in principi attivi e la stessa forma farmaceutica, ma anche lo stesso profilo di efficacia e di tollerabilità del prodotto "innovatore"; in altri termini, deve anche poter vantare di essere un "equivalente terapeutico".

L'equivalenza terapeutica tra due prodotti può, anzi normalmente deve, essere dimostrata mediante ricerche cliniche controllate comparative, effettuate con opportuni disegni sperimentali, direttamente su gruppi di pazienti.

Nel caso di prodotti "generici" (o di altri prodotti copia essenzialmente simili) la loro equivalenza terapeutica con i prodotti di marchio imitati può essere dimostrata in modo surrogato mediante studi di bioequivalenza.

La prima definizione di bioequivalenza risale al 1977 quando la Food and Drug Administration degli Stati Uniti ha stabilito che "sono prodotti bioequivalenti gli equivalenti farmaceutici o alternative le cui velocità ed estensione di assorbimento non evidenziano una significativa differenza quando somministrati alla stessa dose molare di principio attivo $e$ in simili condizioni sperimentali, sia in dose singola che in dose multipla" [1].

Le Linee Guida della Comunità Europea hanno ripreso sostanzialmente la stessa definizione affermando che "due prodotti medicinali sono bioequivalenti se sono equivalenti farmaceutici o alternative e se la loro biodisponibilità (velocità ed estensione) dopo somministrazione alla stessa dose molare sono simili a tal punto che i loro effetti, sia per quanto riguarda l'efficacia che la sicurezza, sono essenzialmente gli stessi" [2].

Una definizione più moderna di bioequivalenza è stata messa a punto nel cor- 
so del "Drug Information Association Workshop" che si è tenuto a Barcellona nel 1991: "Due prodotti sono considerati bioequivalenti quando le loro biodisponibilità derivanti dalla stessa dose molare sono così simili tanto che è improbabile che producano differenze rilevanti negli effetti terapeutici elo avversi" [3].

Una definizione molto simile è quella concordata durante la $\mathrm{I}^{\circ}$ Sessione del Convegno "Bio-International 2", tenutosi a Monaco nel giugno 1994: "Due prodotti farmaceutici sono considerati essere equivalenti quando i loro profili concentrazione-tempo, conseguenti dalla stessa dose molare, sono così simili che è improbabile producano differenze rilevanti negli effetti terapeutici elo avversi" [4].

Gli studi di bioequivalenza non utilizzano parametri clinici di efficacia, ma si limitano a confrontare la biodisponibilità sistemica di due prodotti. I test di bioequivalenza sono, quindi, basati sul confronto statistico di parametri farmacocinetici che caratterizzano la biodisponibilità dei due prodotti: generalmente vengono usati i parametri AUC, Cmax e tmax, ma quando ciò non è fattibile si può ricorrere a parametri relativi all'escrezione urinaria o a

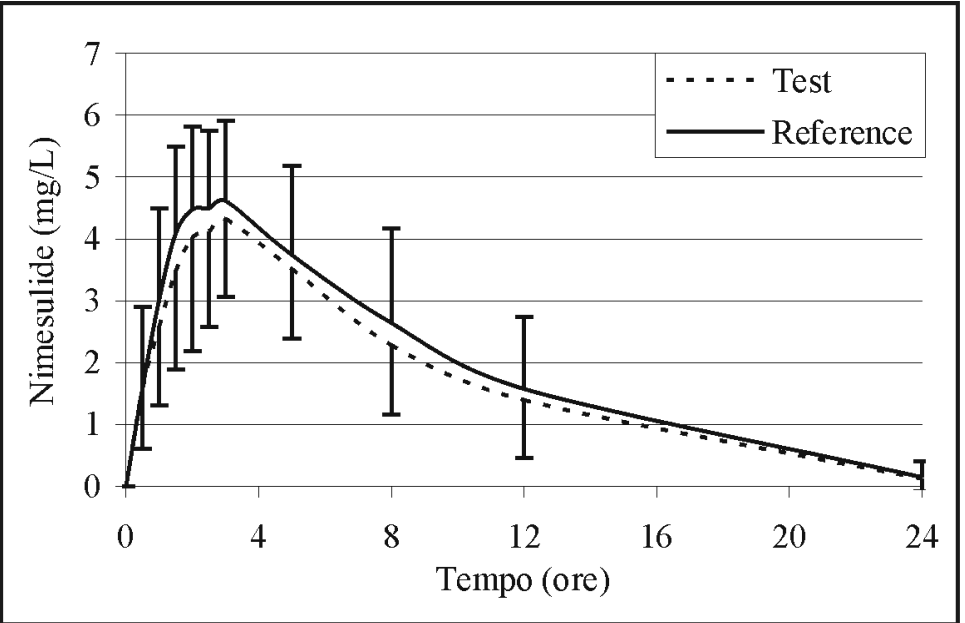

\begin{tabular}{lcc} 
& Reference (Aulin) & Test (generico) \\
\hline $\mathrm{AUC}_{0 \text {-last, }}(\mathrm{min} * \mu \mathrm{g} / \mathrm{mL})$ & $2482,03 \pm 1230,9$ & $2300,52 \pm 1147,9$ \\
$\mathrm{Cmax}(\mu \mathrm{g} / \mathrm{mL})$ & $5,19 \pm 1,27$ & $4,79 \pm 1,59$ \\
$\operatorname{Tmax}(\mathrm{min})$ & $143,7 \pm 59,9$ & $168,7 \pm 69,5$ \\
MRT (min) & $347,4 \pm 98,0$ & $351,7 \pm 89,6$
\end{tabular}

\section{Figura 2}

Esempio di studio di biodisponibilità relativa e di bioequivalenza: curve delle concentrazioni plasmatiche medie $( \pm S D$ ) di nimesulide misurate in un gruppo di 24 soggetti volontari sani dopo singola dose orale $(200 \mathrm{mg}$ ) a digiuno di un prodotto Test (generico) e di un prodotto Reference (Aulin), somministrate in due periodi successivi secondo un disegno di cross-over bilanciato delle sequenza [15] parametri farmacodinamici direttamente correlabili con l'esposizione al farmaco.

La logica decisionale, e la relativa statistica utilizzata nei test di bioequivalenza, è quella di accettare che due prodotti farmaceutici possano generare risultati terapeutici praticamente sovrapponibili nonostante il loro profilo di biodisponibilità presenti differenze rilevabili. Una formulazione test e una formulazione standard sono definite bioequivalenti se si può determinare, con un buon livello di confidenza, che la differenza tra le loro biodisponibilità oscilla entro un range predefinito in modo convenzionale come "intervallo accettabile" di bioequivalenza.

In altre parole, bisogna dimostrare che le differenze di biodisponibilità, che inevitabilmente esistono tra due prodotti essenzialmente simili, non superano un certo range ritenuto compatibile con l'equivalenza terapeutica.

Come si può ben comprendere, si tratta di una logica decisionale e statistica diversa e, per certi aspetti, opposta a quella tradizionale attuata per dimostrare che due prodotti sono significativamente differenti.

Esiste un sostanziale consenso internazionale nel definire un unico intervallo accettabile di bioequivalenza, indipendentemente dal parametro farmacocinetico considerato per confrontare la biodisponibilità del prodotto test con quella del prodotto standard. Tale intervallo è fissato nel range $0,80-1,25$, quando si considera la media dei rapporti individuali tra AUC della formulazione test e AUC della formulazione standard, oppure è fissato entro il range $\pm 0,20$, quando si utilizza la differenza tra parametri normalizzata per il parametro della formulazione standard; il livello di confidenza è generalmente fissato al $90 \%$.

La metodologia statistica per testare la bioequivalenza di due prodotti è stata sviluppata soprattutto negli ultimi due decenni ed è ritenuta adeguata [5-14].

L'intervallo di bioequivalenza è uno standard stabilito convenzionalmente avendo presente soprattutto la variabilità del comportamento in vivo della formulazione piuttosto che la variabilità della risposta terapeutica nella popolazione dei pazienti.

Un intervallo di bioequivalenza così ampio e, soprattutto, non differenziato per categoria terapeutica e per classe farmacologica, tende a trascurare le altre variabili farmacologiche e cliniche che possono incidere significativamente sulla equivalenza terapeutica di due prodotti e potrebbe essere talvolta inadeguato a garantire con sufficiente affidabilità che due prodotti giudicati bioequivalenti siano anche terapeuticamente equivalenti quando 
usati in una popolazione reale di pazienti. Al contrario, in alcuni casi l'intervallo di bioequivalenza potrebbe rivelarsi troppo stretto tanto da indurre in errore e portare ad escludere che due prodotti siano terapeuticamente equivalenti, mentre in realtà lo sono quando applicati in una popolazione reale di pazienti.

Nella figura 2 viene riportato un esempio di studio di bioequivalenza tra un generico di nimesulide e lo standard innovatore (Aulin) [15].

Lo studio della bioequivalenza, o equivalenza farmacocinetica, viene considerato importante sia come misura in vivo della qualità di una nuova forma farmaceutica ad uso sistemico, sia come stima surrogata della sua equivalenza terapeutica con la formulazione di riferimento al fine di fornire al medico e ad altri decisori garanzie circa la sua prescrivibilità e la eventuale intercambiabilità [5].

Gli studi di bioequivalenza sono, infatti, richiesti fondamentalmente in tre diverse situazioni:

1. quando si deve testare una formulazione di un "generico" (prodotto copia) contro un prodotto innovatore;

2. quando si propone l'uso di una forma farmaceutica differente da quella usata negli studi clinici fondamentali;

3. quando si introducono modificazioni significative nelle procedure di fabbricazione della formulazione in commercio.

Gli studi di bioequivalenza sono considerati come il collegamento cruciale tra le formulazioni messe in commercio e quelle usate durante gli studi clinici che hanno portato alla registrazione del prodotto farmaceutico. Infatti, è stato dimostrato che, nel 59\% dei casi, le formulazioni orali approvate dalla FDA (USA) nel periodo gennaio 1981- dicembre 1990 erano differenti da quelle utilizzate nel corso dei trials clinici fondamentali [14].

Va altresì ricordato che lo scostamento medio di biodisponibilità rispetto all'originatore dei generici entrati in commercio negli USA è risultato essere solo del $3,5 \%$ e che lo scostamento di farmacocinetica tra due lotti di uno stesso brand prodotti dalla stessa casa madre non è mai comunque pari a zero [16].

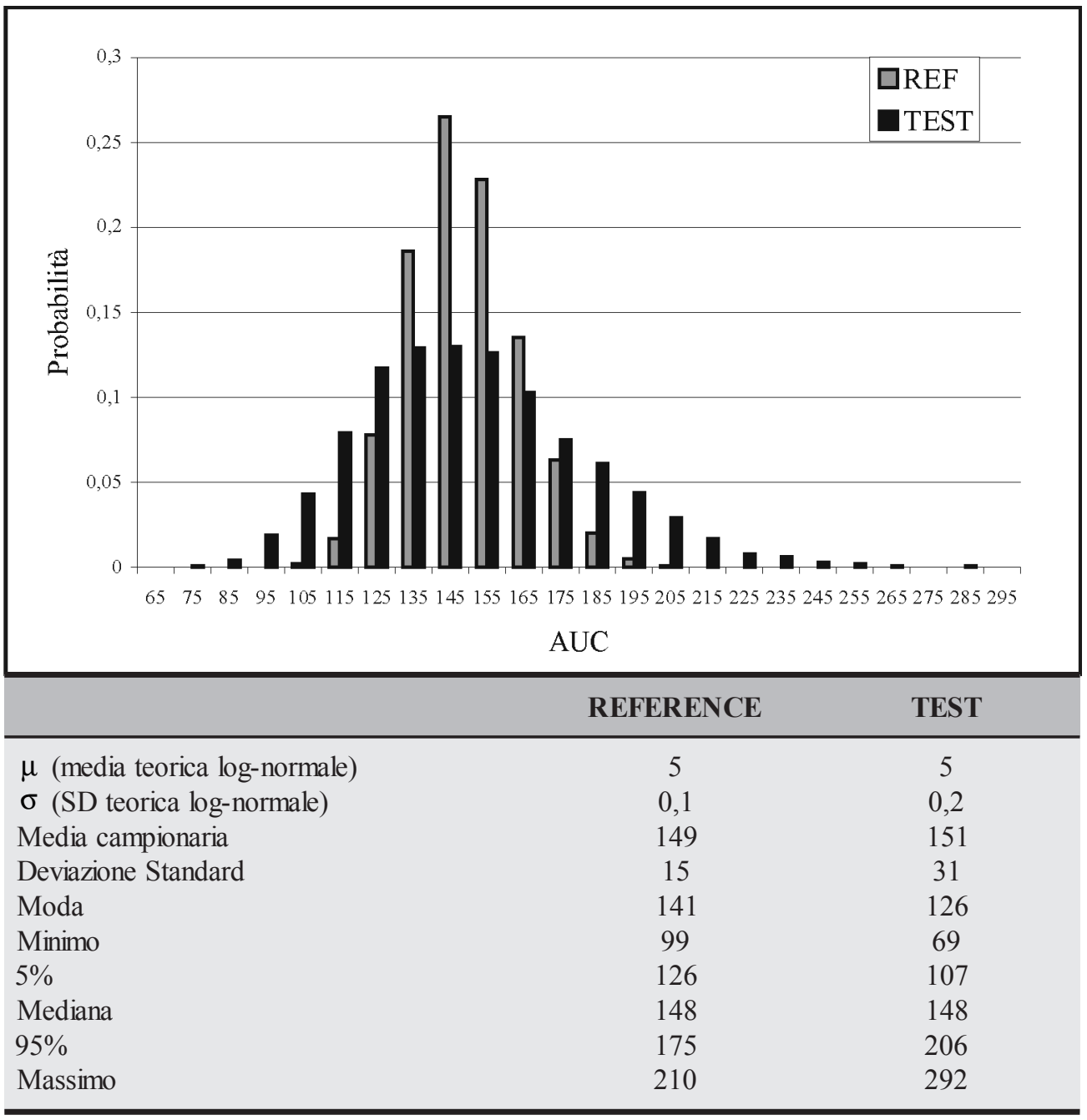

Figura 3

Esempio di

bioequivalenza media in assenza di bioequivalenza di popolazione a causa della differente varianza attorno alla media dei valori delle AUC relative ai due prodotti confrontati. I dati sono stati simulati campionando con il metodo Monte Carlo i valori delle AUC da due distribuzioni log-normali aventi la stessa media e differente deviazione standard 


\section{BIOEQUIVALENZAMEDIA, DIPOPOLAZIONEEDINDIVIDUALE}

L'approfondimento del valore e dell'utilità degli studi di bioequivalenza come stima surrogata della equivalenza terapeutica ha fatto emergere l'esigenza e l'opportunità di dover definire e distinguere tre diversi tipi di bioequivalenza che richiedono procedure e metodi di valutazione differenti: la bioequivalenza media, la bioequivalenza di popolazione e la bioequivalenza individuale [18-20].

Il modello base di bioequivalenza è quello della "bioequivalenza media", definita dal confronto tra le medie dei parametri di biodisponibilità stimati sul prodotto test e quelle stimate sul prodotto standard.

Questo criterio è adatto a verificare le caratteristiche biofarmaceutiche e la qualità media di un prodotto farmaceutico in confronto ad un altro di riferimento. Infatti, l'intervallo di bioequivalenza è uno standard sostanzialmente uniforme per ogni categoria terapeutica stabilito convenzionalmente avendo presente soprattutto la variabilità del comportamento in vivo della formulazione piuttosto che la variabilità della risposta terapeutica nella popolazione dei pazienti.

La stima della bioequivalenza media, tuttavia, non considera la varianza attorno ai valori medi dei parametri di biodisponibilità e non valuta la equivalenza della risposta alle due formulazioni nei singoli soggetti [21].

Per rimediare a queste carenze, Anderson e Hauck introdussero, prima, il concetto di "bioequivalenza di popolazione" come generalizzazione della bioequivalenza media e, poi, il concetto di "bioequivalenza individuale" [18-20].

Due formulazioni possono essere considerate "bioequivalenti per una popolazione" se, oltre al valore medio dei parametri di biodisponibilità, anche le loro distribuzioni attorno alle medie sono sufficientemente simili. Le AUC o le Cmax di due formulazioni possono avere un valore medio sufficientemente simile, ma una varianza significativamente differente (vedi figura 3). In tal caso le due formulazioni non sono equivalenti per la popolazione perché le distribuzioni delle loro biodisponibilità sono significativamente differenti.

Dimostrare la bioequivalenza di popolazione assume un'importanza rilevante per poter assicurare il medico che può attendersi un risultato terapeutico mediamente equivalente nella popolazione dei suoi pazienti, se inizia un nuovo trattamento con un farmaco generico piuttosto che con l'equivalente prodotto di marchio.

La bioequivalenza di popolazione, tuttavia, non fornisce alcuna informazione circa la probabilità che la risposta del singolo paziente a due formulazioni sia equivalente.

Per poter fare questa previsione occorre stimare la bioequivalenza individuale, ossia la bioequivalenza entro soggetto, e valutare in quale percentuale i singoli soggetti rispondono in modo equivalente al prodotto generico $\mathrm{e}$ al prodotto innovatore.

Sono stati proposti diversi metodi per risolvere il problema della biodisponibilità individuale. La FDA ha proposto la regola del 75/ 75 (almeno il $75 \%$ dei soggetti testati devono avere un rapporto individuale di biodisponibilità entro il range 75-125\%). Altri autori hanno proposto metodi più rigorosi dal punto di vista statistico, ma analoghi come logica decisionale [18]. Il TIER (Test of Individual Equivalence Ratios) di Anderson e Hauck richiede venga fissata la proporzione minima di soggetti di una popolazione target per cui le due formulazioni testate debbano essere equivalenti. Questa regola viene riassunta nel numero minimo di soggetti con un rapporto individuale di biodisponibilità entro il range accettabile di bioequivalenza necessario per concludere per la equivalenza in relazione alla dimensione del campione e al livello di errore di tipo I. Viene anche raccomandato di calcolare $\mathrm{i}$ limiti fiduciali di tale proporzione.

La tabella 3 riporta un caso simulato di bioequivalenza messo a punto per illustrare l'importanza della bioequivalenza individuale e per dimostrare la possibilità che un test positivo di bioequivalenza media e di popolazione non riveli l'assenza della bioequivalenza individuale in un numero eccessivo di soggetti. Sono state simulate, mediante il metodo Monte Carlo, due serie di 30 valori di AUC, partendo da una medesima distribuzione log-normale. L'abbinamento casuale, uno a uno, dei valori delle AUC di una serie con quelli dell'altra serie per ricostruire possibili sequenze di trattamento intrasoggetto, ha consentito di verificare che talvolta si possono realizzare condizioni di non-bioequivalenza individuale in un numero elevato di soggetti, tanto da dover concludere che i due prodotti, pur essendo bioequivalenti come valore medio e di popolazione, non rispettano i criteri della bioequivalenza individuale.

La biodisponibilità individuale si configura, dunque, come il criterio fondamentale per poter applicare la norma della sostituibilità tra formulazioni nel corso di un trattamento in atto, 
senza pregiudicare il profilo terapeutico e di sicurezza ottenuti con la prima formulazione [22].

Purtroppo, il disegno della quasi totalità degli studi di bioequivalenza non consente di stimare la bioequivalenza individuale, ma solo la bioequivalenza media e/o la bioequivalenza di popolazione. Pertanto i farmaci generici attualmente in commercio in Italia non possono garantire un livello adeguato di bioequivalenza individuale, ma solo un livello adeguato di bioequivalenza media a livello di popolazione.

In queste condizioni la sostituzione automatica di un farmaco di marchio attualmente in uso con un farmaco generico o tra due generi- ci, soprattutto se effettuata dal farmacista senza il coinvolgimento della supervisione del medico curante, può comportare qualche problema per alcuni pazienti nei confronti dei quali i due prodotti, quello in uso e il generico che lo sostituisce, possono non essere equivalenti come efficacia e come tollerabilità [23-30].

Questo problema, ben noto in letteratura, risulta più delicato quando sono coinvolti farmaci d'uso cronico dotati di scarsa maneggevolezza e di basso indice terapeutico, come gli antiepilettici, gli antiaritmici e gli anticoagulanti.

La sostituzione del prodotto di marchio con un prodotto generico è tuttavia praticabile con

\begin{tabular}{|c|c|c|c|c|c|c|c|}
\hline \multirow{2}{*}{\multicolumn{2}{|c|}{ Soggetto $\mathbf{N}^{\circ}$}} & \multicolumn{3}{|c|}{$\begin{array}{l}\mathrm{I}^{\circ} \text { Combinazione AUC } \\
\text { intrasoggetto }\end{array}$} & \multicolumn{3}{|c|}{$\begin{array}{l}\text { II }^{\circ} \text { Combinazione AUC } \\
\text { intrasoggetto }\end{array}$} \\
\hline & & TEST & REF & TEST/REF & TEST & REF & TEST/REF \\
\hline & 1 & 152 & 160 & 0,95 & 152 & 160 & 0,95 \\
\hline & 2 & 150 & 152 & 0,98 & 150 & 152 & 0,98 \\
\hline & 3 & 155 & 161 & 0,97 & 155 & 161 & 0,97 \\
\hline & 4 & 154 & 140 & 1,10 & 154 & 140 & 1,10 \\
\hline & 5 & 140 & 134 & 1,05 & 140 & 134 & 1,05 \\
\hline & 6 & 134 & 148 & 0,91 & 134 & 148 & 0,91 \\
\hline & 7 & 148 & 140 & 1,06 & 148 & 140 & 1,06 \\
\hline & 8 & 124 & 143 & 0,87 & 124 & 143 & 0,87 \\
\hline & 9 & 159 & 164 & 0,97 & 159 & 164 & 0,97 \\
\hline & 10 & 177 & 149 & 1,19 & 161 & 149 & 1,08 \\
\hline & 11 & 172 & 162 & 1,06 & 172 & 132 & 1,30 \\
\hline & 12 & 160 & 170 & 0,94 & 160 & 170 & 0,94 \\
\hline & 13 & 161 & 141 & 1,14 & 177 & 141 & 1,26 \\
\hline & 14 & 132 & 160 & 0,82 & 132 & 160 & 0,82 \\
\hline & 15 & 143 & 144 & 0,99 & 143 & 144 & 0,99 \\
\hline & 16 & 148 & 181 & 0,82 & 140 & 181 & 0,77 \\
\hline & 17 & 149 & 166 & 0,90 & 149 & 158 & 0,95 \\
\hline & 18 & 163 & 126 & 1,29 & 163 & 126 & 1,29 \\
\hline & 19 & 128 & 170 & 0,76 & 128 & 170 & 0,76 \\
\hline & 20 & 137 & 154 & 0,89 & 137 & 154 & 0,89 \\
\hline & 21 & 152 & 155 & 0,98 & 152 & 155 & 0,98 \\
\hline & 22 & 142 & 130 & 1,09 & 142 & 130 & 1,09 \\
\hline & 23 & 142 & 132 & 1,07 & 142 & 162 & 0,87 \\
\hline & 24 & 163 & 185 & 0,88 & 133 & 185 & 0,72 \\
\hline & 25 & 120 & 155 & 0,77 & 120 & 155 & 0,77 \\
\hline & 26 & 182 & 156 & 1,16 & 182 & 156 & 1,16 \\
\hline & 27 & 133 & 123 & 1,09 & 163 & 123 & 1,33 \\
\hline & 28 & 135 & 142 & 0,95 & 135 & 142 & 0,95 \\
\hline & 29 & 129 & 158 & 0,81 & 129 & 166 & 0,78 \\
\hline & 30 & 140 & 155 & 0,90 & 148 & 155 & 0,95 \\
\hline Media & & 148 & 152 & 0,98 & 148 & 152 & 0,98 \\
\hline SD & & 15 & 15 & 0,13 & 15 & 15 & 0,16 \\
\hline Min & & 120 & 123 & 0,76 & 120 & 123 & 0,72 \\
\hline Mediana & & 148 & 154 & 0,97 & 148 & 154 & 0,96 \\
\hline Max & & 182 & 185 & 1,29 & 182 & 185 & 1,33 \\
\hline
\end{tabular}

\section{Tabella 3}

Dimostrazione della possibilità che due prodotti mediamente bioequivalenti possano essere non

bioequivalenti in un numero variabile di soggetti. Nel caso della $I^{\circ}$ combinazione di AUC intrasoggetti, il rapporto AUC TEST/AUC $R E F$ supera il range di bioequivalenza $(0,80$ $1,25)$ in 3 soli soggetti, cioè in una percentuale bassa (10\%) che consente di concludere per la bioequivalenza individuale; nel caso della $I I^{\circ}$ combinazione, invece, tale rapporto tra AUC supera il range di bioequivalenza in 9 soggetti, una percentuale elevata $(30 \%)$ che fa decidere per una non bioequivalenza individuale 
sufficienti margini di affidabilità e sicurezza per la maggior parte delle altre classi di farmaci dotati per lo più di ampi margini di manegevolezza.

È importante evidenziare come la dimostrazione di bioequivalenza non possa essere estrapolata al di fuori dei prodotti direttamente confrontati e come, pertanto, non possa essere considerata documentata per estrapolazione la bioequivalenza tra un prodotto generico ed eventuali altri standard di riferimento o tra generici diversi di uno stesso innovatore. In altri termini, in questo campo non può essere applicata la logica transitiva per cui se il prodotto $\mathrm{A}$ è equivalente al prodotto $\mathrm{B}$ e il prodotto $\mathrm{B}$ è equivalente al prodotto $C$ ne consegue che anche il prodotto A deve essere ritenuto equivalente al prodotto $C$. Infatti, nel caso in cui la differenza di biodisponibilità tra $\mathrm{A}$ e $\mathrm{B}$ fosse $+15 \%$ e quella tra $\mathrm{B}$ e $\mathrm{C}-15 \%$, la differenza di biodisponibilità tra $\mathrm{A} \mathrm{e} \mathrm{C}$ sarebbe del $30 \% \mathrm{e}$ quindi $\mathrm{A}$ e $\mathrm{C}$ non potrebbero essere dichiarati bioequivalenti (figura 4).

Questo problema è stato affrontato negli Stati Uniti e risolto con la pubblicazione di un libro (Orange Book), aggiornato periodicamente da una commissione tecnica della FDA, nel quale, per ogni generico in commercio, sono riportate sistematicamente tutte le sostituzioni approvate ed ammissibili, perché documentate [31].

Precisati gli aspetti problematici intrinseci alla categoria dei farmaci "generici" è importante rassicurare gli utenti e gli operatori sanitari, sottolineando ancora una volta che si tratta di prodotti medicinali registrati dal Ministero della Salute e pertanto sottoposti ad un vaglio tecnico analogo a quello di tutte le altre specialità medicinali.

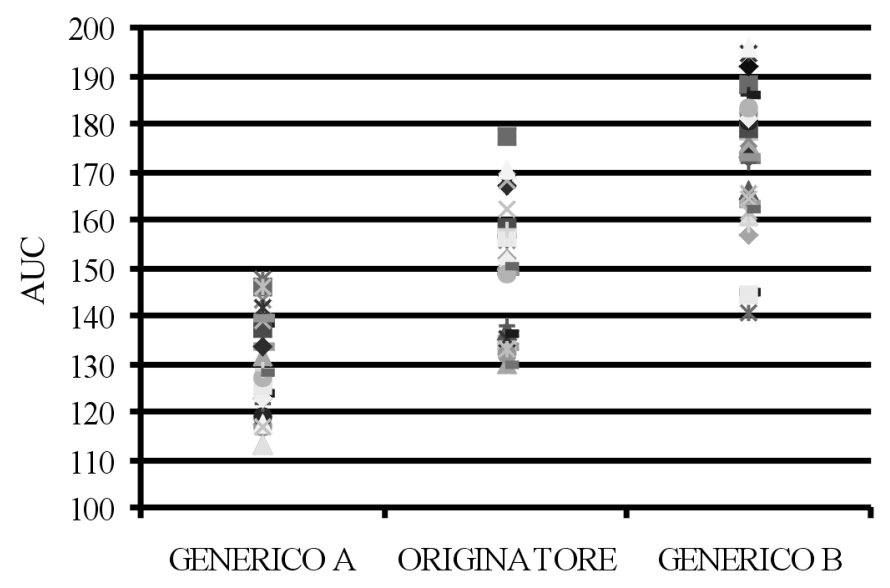

Figura 4

Non-bioequivalenza fra due generici, ciascuno bioequivalente con il farmaco innovatore
Occorre anche informare che il Ministero della Salute ha il potere di controllo su ogni tappa del processo di ricerca, sviluppo e produzione dei farmaci, compresi i generici. In particolare, presso il Ministero della Salute esiste un apposito Ufficio deputato alle ispezioni dei Centri di Ricerca che effettuano gli studi di bioequivalenza e degli impianti di produzione dei medicinali.

Il prodotto "generico" ha quindi piena dignità e offre gli stessi livelli di sicurezza garantiti per tutti i farmaci registrati.

\section{CONSIDERAZIONI FARMACOECONOMICHE}

L'impatto prevalente del farmaco generico sul sistema sanitario avviene in ambito economico. Infatti, il farmaco generico non riveste alcun interesse particolare sul piano terapeutico, non essendo un prodotto innovativo e non comportando significative modificazioni dei livelli di efficacia e tollerabilità, mentre la sua disponibilità e diffusione sul mercato sono fattori considerati importanti per: 1. favorire un mercato farmaceutico maggiormente aperto alla concorrenza;

2. innescare una dinamica di riduzione dei prezzi di molti medicinali;

3. consentire significativi risparmi sulla spesa farmaceutica pubblica e quindi liberare risorse da destinare ai farmaci innovativi più costosi.

Questi obiettivi ideali sono realizzati in modo imperfetto e incompleto in tutti i paesi industrializzati che hanno sviluppato un mercato dei generici.

La dinamica della concorrenza e dei prezzi varia molto nei diversi paesi in funzione della struttura locale dell'industria farmaceutica, delle strategie di marketing delle multinazionali e delle aziende genericiste, delle politiche adottate per controllare la spesa farmaceutica, nonché delle normative specifiche riguardanti la formazione del prezzo e la distribuzione dei farmaci generici.

In alcune nazioni le multinazionali sono riuscite a riprendere il totale controllo sul mercato farmaceutico, creando e gestendo esse stesse il settore dei generici ed adottando strategie di mercato che in alcuni casi ha portato ad un aumento dei prezzi dei prodotti di marchio a fronte di una riduzione del prezzo dei corrispondenti generici.

Un recente studio ha dimostrato come la politica della formazione dei prezzi dei generici, adottata all'inizio degli anni '90 nell'Ontario in Canada, non solo abbia fallito l'obiettivo di abbassare il prezzo d'acquisto dei farmaci ma abbia anche comportato un risultato opposto. 
Infatti i prezzi d'acquisto dei generici si sono localizzati praticamente tutti vicini al valore massimo consentito (70\% del prezzo del prodotto di marchio per il primo generico introdotto; $90 \%$ del prezzo del primo generico per i successivi ingressi sul mercato di analoghi generici), senza ulteriori dinamiche di riduzione su base concorrenziale [32].

In Italia il mercato dei generici è iniziato praticamente da meno di 2 anni ed ha rivelato una dinamica di riduzione dei prezzi su base concorrenziale discretamente attiva, comportando per alcuni prodotti un abbattimento del prezzo iniziale di oltre il $50 \%$.

Occorre evidenziare come una eccessiva riduzione dei prezzi non sempre possa essere considerata un fatto positivo e frutto di una sana concorrenza. Infatti, una caduta troppo forte dei prezzi suscita perplessità, crea sfiducia nei farmaci in generale, e nei farmaci generici in particolare, e potrebbe essere il risultato di un abbassamento dei livelli qualitativi del prodotto. Per evitare queste conseguenze negative, proteggere l'utente e garantire un livello qualitativo elevato in un contesto di competitività di mercato, sarebbe opportuno introdurre non solo un prezzo massimo consentito ma anche un prezzo minimo consentito per i farmaci generici.

Il mercato dei generici ha raggiunto quote consistenti (alcune decine di punti percentuali) in alcuni paesi come gli Stati Uniti, il Regno Unito, la Germania e l'Olanda. In Italia è ancora un mercato marginale che rappresenta poco più del $2 \%$ in volume e poco più dell' $1 \%$ in valore sul mercato farmaceutico totale. È tuttavia un mercato in lenta espansione sia come introduzione di nuovi prodotti sia come volume complessivo di confezioni vendute. Il numero di prodotti generici disponibile è relativamente elevato, ma tipicamente polarizzato attorno alle molecole che possono ottenere un prezzo maggiormente remunerativo o che hanno un vasto mercato.

La figura 5 riporta le quote percentuali di mercato, in volume e valori, dei primi venti farmaci più venduti come generico in Italia. Nel 2002 solo i generici della ticlopidina hanno ottenuto una quota di mercato superiore al $50 \%$ del totale, mentre tutti gli altri hanno raggiunto quote percentuali nettamente inferiori.

D'altra parte le quote complessive di mercato dei generici rispetto al loro potenziale massimo (genericato) è nettamente inferiore al $10 \%$ e varia sensibilmente nelle diverse Regioni italiane, come evidenzia la figura 6 . Nel 2001 solo la Toscana aveva raggiunto il 9\% del mercato dei generici sul genericato, mentre tutte le altre Regioni si collocavano a quote inferiori al $5 \%$.
Il mercato dei generici in Italia ha, dunque, ancora un grande potenziale di sviluppo.

Per poter cogliere i benefici di tale mercato potenziale occorre, tuttavia, creare un clima di fiducia in questa tipologia di prodotto farmaceutico sia negli operatori sanitari, medici farmacisti e infermieri, sia nella popolazione dei pazienti. L'incentivo del risparmio è importante, ma, evidentemente, altri forti condizionamenti prevalgono ancora nell'orientare verso la scelta del generico.

Personalmente ritengo che la norma della sostituibilità del generico e i sistemi di pressione sulla classe medica perché prescrivano i generici, pur importanti, siano strumenti che producono effetti solo marginali, come dimostrano le basse quote di mercato finora raggiunte.

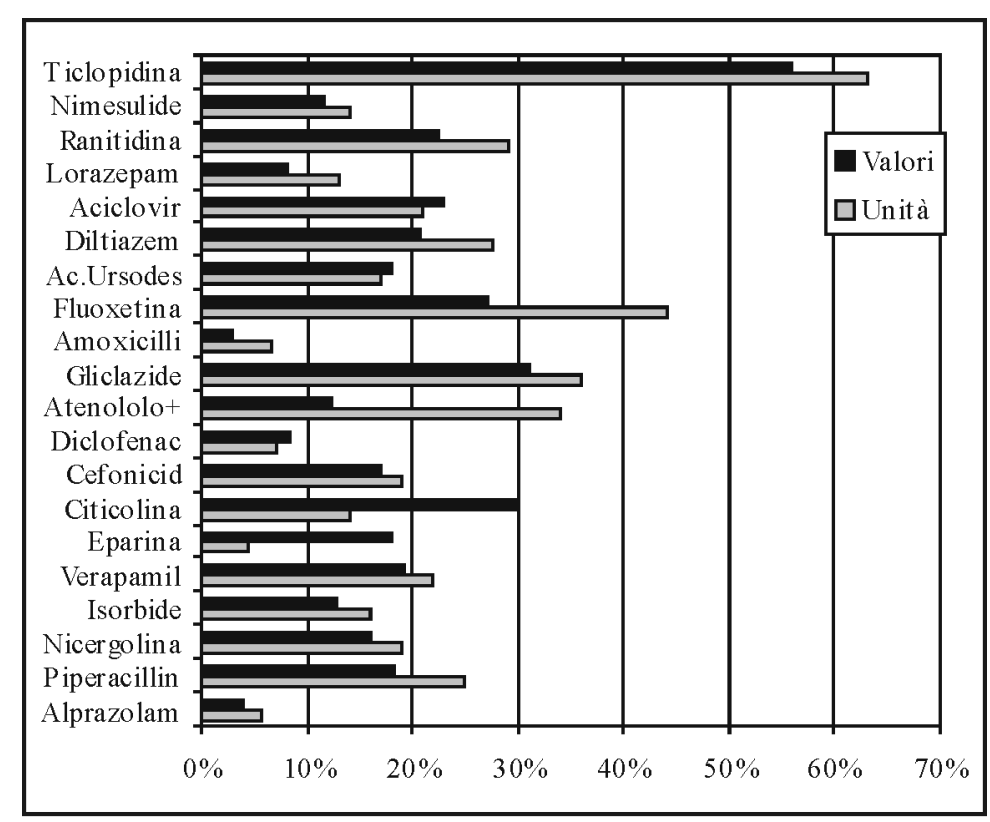

Figura 5

Market share \% dei primi 20 generici più venduti in Italia (IMS 2002)

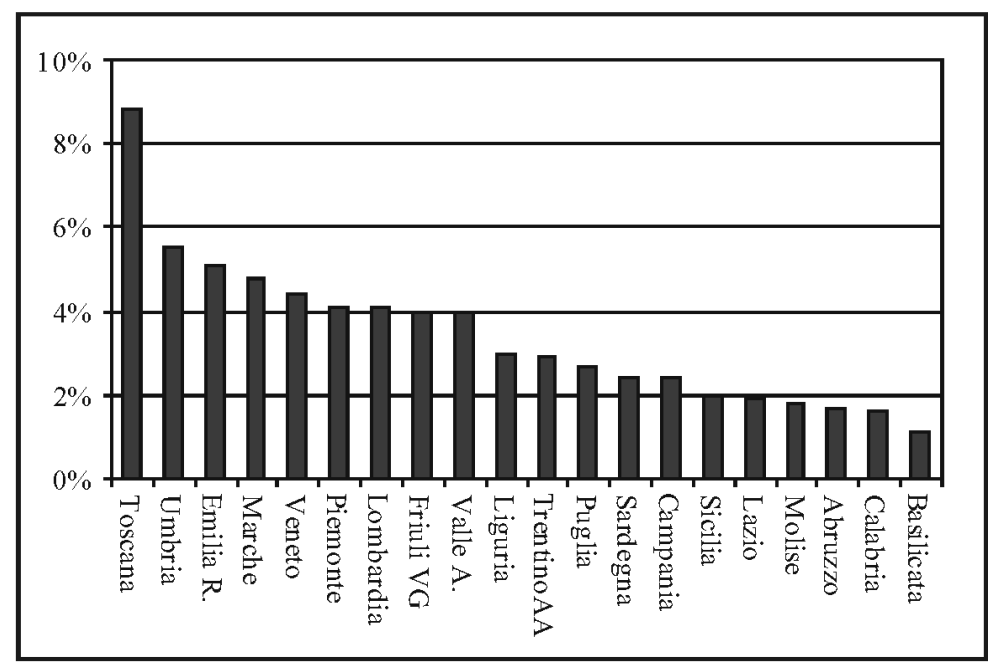

Figura 6

Incidenza percentuale di mercato del generico sul genericato nelle diverse regioni italiane (dati IMS 2001) 
L'immagine positiva del generico può essere data in modo decisivo dalla stessa azienda produttrice di generici che, attraverso lo sviluppo galenico su basi tecnologiche moderne, sappia proporre sul mercato prodotti di alta qualità ad un prezzo concorrenziale.
Sotto questa prospettiva, il mercato del farmaco generico può rappresentare un'importante opportunità di sviluppo per l'industria farmaceutica italiana ed un'importante fattore di razionalizzazione della spesa farmaceutica pubblica e privata.

\section{BIBLIOGRAFIA}

1. Food and Drug Administration. Bioavailability and Bioequivalence requirements. US FDA Fed. Reg. 42: 1642-1653 (1977).

2. Rauws AG. Bioequivalence: an European Community regulatory perspective. In: Pharmaceutical bioequivalence, Swarbrick J. (ed.) Drugs Pharm. Sci., vol.. 48, M. Dekker Inc., New York (1991).

3. Drug Information Association. International Harmonization and Consensus DIA Meeting. Drug Information J., 25: 471-482 (1991).

4. Midha KK, Rauws AG et al. Session I: Bioequivalence: Quality control and therapeutic Surragate? In: Midha KK, Blume HH. (eds.): Bio-International 2. Bioavailability, Bioequivalence and Pharmacokinetics. Medpharm Scientific Publications, Stuttgart, pp. 16-17 (1995).

5. Midha KK, Hubbard JW. Aims and consequences of bioequivalence studies. In: Midha KK, Blume HH. (eds.): Bio-International 2. Bioavailability, Bioequivalence and Pharmacokinetics. Medpharm Scientific Publications, Stuttgart, pp.29-34 (1995).

6. Westlake WJ. Use of confidence intervals in analysis of comparative bioavailability trials. J. Pharm. Sci. 61: 1340-1341 (1972)

7. Westlake WJ. Symmetric confidence intervals for bioequivalence trials. Biometrics 30: 319-327 (1974)

8. Mantel N. Do we want confidence intervals symmetrical about the null value (letter). Biometrics 33. $759-760$ (1977)

9. Kirkwood TBL. Bioequivalence testing - A need to rethink. Biometrics 37: 589-594 (1981)

10. Mandallaz D, Mau J. Comparison of different methods for decision-making in bioequivalence assessment. Biometrics 37: 213$222(1981)$

11. Schuirman DJ. A comparison of the two one-sided tests procedure and the power approach for assessing the equivalence of average bioavailability. J. Pharmacokin. Biopharm. 15: 657-680 (1987)

12. Hauschke D, Steinijans VW. A distribution-free procedure for the statistical analysis of bioequivalence studies. Int. J. Clin. Pharm. Toxicol. 28: 72-80 (1990)

13. Rescigno A. Bioequivalence. Pharm. Res. 9: 925-928 (1992).

14. Benet LZ. Bioavailability and bioequivalence: definitions and difficulties in acceptance criteria. In: Midha KK., Blume HH. (eds.): Bio-International. Bioavailability, Bioequivalence and Pharmacokinetics. Medpharm Scientific Publications, Stuttgart, pp.27-35 (1993).

15. Eandi M, Della Pepa C. Biodisponibilità relativa e bioequivalenza di un prodotto generico di nimesulide versus innovatore. (File personale)

16. Murphy JE. Generic substitution and optimal patient care, Arch Intern Med/Vol 159,429-433,1999

17. Rheinstein PH. Therapeutic inequivalence. Drug Saf. 1990; 5 (Suppl 1): 114-9 (1990)

18. Anderson S, Hauck WW. Consideration of individual bioequivalence. J. Pharmacok. Biopharm. 18: 259-273 (1990).

19. Hauck WW, Anderson S. Individual bioequivalence: what matters to the patient. Stat-Med. 1991 Jun; 10(6): 959; discussion 95960

20. Hauck WW, Anderson S. Individual vs. Average Bioequivalence: when is average not sufficient? In: Midha KK, Blume HH. (eds.): Bio-International 2. Bioavailability, Bioequivalence and Pharmacokinetics. Medpharm Scientific Publications, Stuttgart, pp.87-90 (1995).

21. Lasagna L. Efficacy and generic drugs (letter). Lancet 2: 218-219, 1987.

22. Nation RL, Sansom LN. Bioequivalence requirements for generic products. Pharmacol. Ther. 62: 41-55 (1994)

23. Welty TE, Pickering PR, Hale BC et al. Loss of seizure control associated with generic substitution of carbamazepine. Annal Pharmacother 26: 775-777, 1992.

24. Hendeles L, Hochhaus G, Kazerounian S. Generic and alternative brand-name pharmaceutical equivalents: select with caution. Am-J-Hosp-Pharm. 1993 Feb; 50(2): 323-9

25. Bell K. Prescription drugs: the problem with substitution. Aus Fam Physician 23: 2373, 1994.

26. Schnur W. Potential hazards of generic antidepressants in the elderly. Am Fam Phsician 51: 48, 1995.

27. Besag FMC. Is generic prescribing acceptable in epilepsy? Drug Saf 23: 173, 2000.

28. Reiffel JA. Formulation substitution and other pharmacokinetic variability: unappreciated variables affecting antiarrhythmic efficacy and safety in clinical practice. Am J Cardiol 85: 46D, 2000.

29. Hope KA, Havrda DE. Subtherapeutic INR values associated with a switch to a generic warfarin. Ann Pharmacother 35: 183, 2001.

30. Lesser RP, Krauss G. Buy some today: Can generics be safely substituted for brand-name drugs? Neurology 57: 571-573, 2001.

31. Food and Drug administration: Center for Drug Evaluation and Research. Orange Book, $22^{\text {nd }}$ Ed. www.fda.gov.

32. Anis AH, Guh DP, Woolcott J. Lowering generic drug prices: Less regulation equals more competition. Med Care 41: 135-141, 2003. 